Alcibiades Bustamante Valdivia ${ }^{1,2}$

Liz Caballero Cartagena ${ }^{1}$

Natalia Enciso Sarria ${ }^{1}$

Ingrid Salazar Távara ${ }^{1}$

André F. Teixeira e Seabra ${ }^{2}$

Rui Manuel Garganta da Silva²

José António Ribeiro Maia²

\title{
COORDINACIÓN MOTORA: INFLUENCIA DE LA EDAD, SEXO, ESTATUS SOCIO-ECONÓMICO Y NIVELES DE ADIPOSIDAD EN NIÑOS PERUANOS
}

\author{
MOTOR COORDINATION: INFLUENCE OF AGE, SEX, SOCIO-ECONOMIC \\ STATUS AND LEVELS OF ADIPOSITY, IN PERUVIAN CHILDREN
}

\begin{abstract}
RESUMEN
Caracterizar el nivel de la coordinación motora (CoM) y determinar la influencia de la edad, sexo, estatus socioeconómico y de la adiposidad subcutánea en la CoM de escolares peruanos de seis a los once años de edad. El tamaño de la muestra comprendió a 4,007 niños (mujeres= 1889; varones= 2118) de escuelas del área metropolitana de Lima, Perú. La CoM fue determinado a través de la batería de pruebas KTK (Kiphard y Schilling, 1974) que comprende cuatro pruebas: equilibrio a la retaguardia (ER), saltos laterales (SL), saltos monopedales (SM) y transposición lateral (TL). La adiposidad subcutánea fue establecida a partir de la suma de los pliegues cutáneos del tríceps, subescapular y pantorrilla. El estatuto socioeconómico (ESE) fue evaluado según la localización física de cada escuela (tipo de vecindad). Los análisis estadísticos exploratorios, descriptivos e inferenciales (ANOVA II así como modelos lineales de regresión) fueron realizados en SPSS 15. Existen incrementos significativos de valores medios de las pruebas de KTK en ambos sexos y a lo largo de la edad. Los niños con adiposidad elevada presentan rendimientos inferiores en todas las pruebas. Los niños superan a las niñas. EI ESE alto presenta una influencia mayor sólo en la prueba de SM, y el ESE bajo ha demostrado ser relevante en la ejecución de ER. Conclusiones - La CoM es altamente específica a cada género. El nivel de adiposidad presenta una influencia negativa en cada prueba de coordinación, también en la coordinación total. El ESE no parece ser un predictor concluyente de la coordinación motora en niños.
\end{abstract}

Palabras-clave: Coordinación motora; Adiposidad subcutánea; Estatuto socioeconómico.

\section{ABSTRACT}

The objective of this study was to describe motor coordination (CoM) levels, in addition to the influence of age, socioeconomic status (SES), sex and subcutaneous adiposity on the CoM of Peruvian children aged six to eleven years. The sample size was 4007 children ( $n=1889$ females; $n=2118$ males) from several schools in the metropolitan area of Lima, Peru. CoM was assessed with the KTK (Kiphard and Schilling, 1974) test battery that includes 4 tests: balancing backward (BB), hopping on one leg (HO), jumping sideways (JS) and shifting platforms (SP). Subcutaneous adiposity included the sum of several skinfolds: triceps, subscapular and calf. Socioeconomic status (SES) was evaluated according to the physical location of each school (type of neighborhood). Exploratory, descriptive and inferential statistical analyses (ANOVA II as well as regression linear models) were performed using SPSS 15. Significant increases in mean values of CoM were observed in both sexes and across age. Children with high adiposity exhibited lower performance in all tests. Boys outperformed girls. High SES has a greater influence only on $\mathrm{HO}$, and a low SES proved relevant to BB performance. It can be concluded that CoM is highly specific to each gender. Body fat levels have a negative influence on each coordination test, as well as on overall coordination. SES does not seem to be a conclusive predictor of motor coordination in children.

Key words: Motor coordination; Subcutaneous fat; Socioeconomic statute.

1 Universidad Nacional de Educación "Enrique Guzmán y Valle", La Cantuta. Lima, Perú.

2 Laboratório de Cineantropometria e Estatística Aplicada da Faculdade de Desporto da Universidade do Porto,

Porto, Portugal. 


\section{INTRODUCCIÓN}

El estudio de la coordinación motora (CoM) en niños en edad escolar reviste una enorme importancia desde el punto de vista pedagógico, psicomotor y epidemiológico ${ }^{1}$. La capacidad de CoM es un determinante en el progreso educativo de los niños, así como en su desarrollo integral ${ }^{2}$. La enorme complejidad de la CoM en cuanto a su interpretación abarca desde lo puramente observable a los numerosos y complicados mecanismos neuro-fisiológicos que implica. Según Kiphard ${ }^{3}$, por coordinación del movimiento comprendemos la interacción armoniosa y, en lo posible, económica, del sistema neuro-muscular y del sistema perceptivo con el fin de producir acciones cinéticas precisas y equilibradas (motricidad voluntaria) y reacciones rápidas y adaptadas a la situación (motricidad refleja).

Identificar niveles de desarrollo coordinativo alcanzados en diferentes grupos etarios en distintas condiciones socio-económicas y distinguir, con alguna precisión, a los niños con insuficiencia coordinativa han sido preocupaciones de gran relevancia en el contexto educativo ${ }^{4}$. De otro lado, al presentar la CoM una estructura multidimensional y no poder ser medido directamente, es de gran importancia utilizar en las evaluaciones una batería de pruebas que exprese una adecuada operacionalización ${ }^{5}$. Schilling e Kiphard ${ }^{6}$, sobre la base de sus preocupaciones pedagógicas y clínicas, desarrollaron la batería de tests de coordinación corporal para niños (Körperkoordinationstest für Kinder, KTK) para identificar y diagnosticar a niños con dificultades de movimiento y coordinación entre los cinco y los catorce años. Después de varios estudios de análisis factorial exploratoria, identificaron un factor designado como coordinación corporal que contiene las cuatro pruebas actuales de la batería KTK.

En el contexto del presente estudio, es de nuestro interés verificar el grado de desarrollo coordinativo y, sobre todo, analizar la influencia de algunos factores asociados como son la edad, el sexo, el estatus socio-económico (ESE) y la adiposidad en el nivel de coordinación motora en una muestra de niños peruanos que son parte de un país en vías de desarrollo. El desarrollo coordinativo en relación con la edad y el sexo fueron abordados en diferentes estudios, principalmente en países desarrollados, siendo los más referenciados los siguientes: Schilling y Kiphard $^{6}$ hallaron en escolares alemanes la existencia de un incremento lineal en ambos sexos; sin embargo, Willimezik ${ }^{7}$, en un estudio longitudinal (6,7 a los 10,7 años), encontró resultados que reflejaban la existencia de un padrón de desarrollo específico para cada sexo y no un padrón de desarrollo invariante como aseguraban Kiphard y Schilling; ${ }^{6}$ mientras, Kobayashi ${ }^{8}$, en una muestra de niños japoneses de seis a los doce años, verificó diferencias significativas sólo en la edad de siete para los ocho años y no se identificó en las restantes edades. En estudios de naturaleza transversal realizados en Portugal por Andrade ${ }^{9}$ y Gomes $^{5}$, se constató que el desempeño era siempre superior en grupos etarios de edad más avanzada en relación con los de menor edad. Maia y Lopes ${ }^{10}$, en un estudio longitudinal con niños portugueses de ambos sexos (6/7 a los 9/10 años de edad), verificaron el aumento de los desempeños coordinativos a lo largo de la edad, sin encontrar diferencias significativas entre sexos.

En cuanto a la asociación entre composición corporal y coordinación motora, el número de estudios es más escaso. Este hecho no permite establecer con mayor claridad la magnitud de dependencia entre estas dos variables. Graf et al ${ }^{11,12}$ en un estudio caso-control como parte de un programa de intervención primaria que combina la educación en salud y la actividad física en escuelas primarias alemanas, analizaron la influencia del IMC en las capacidades motoras. Encontraron que los valores de la prueba de Saltos Laterales del KTK eran más altos y estadísticamente significativos en niños con IMC normal en relación con los que presentaban sobrepeso y obesidad. Los mismos autores, en otro estudio, encontraron que el cociente motor (CM) de los niños con sobrepeso y obesidad presentaba valores más bajos en relación con los niños con peso normal o bajo peso. Por otro lado, Maia y Lopes ${ }^{13}$ encontraron que los valores de adiposidad presentan una asociación ligera con el nivel de desarrollo coordinativo, observándose una influencia más marcada en los niños; por otro lado, comprobaron que el IMC exhibe una influencia baja a moderada en los resultados de cada prueba del KTK.

En América del Sur podemos señalar los siguientes estudios: Silva ${ }^{14}$, al realizar un estudio con 1000 escolares brasileros de siete a los diez años de edad, verificó la presencia de diferencias significativas en los valores del cociente motor a favor de los niños; Bustamante et al $^{15}$, en una muestra de 3164 escolares peruanos de ambos sexos, pertenecientes a una faja etaria de seis a los once años de edad, reportó que los escolares evidenciaron un incremento de los valores medios a lo largo de la edad. Los niños presentaban mejores desempeños coordinativos en las diferentes pruebas de la batería KTK a excepción de la prueba de Equilibrio a Retaguardia.

La insuficiencia de información acerca del desarrollo coordinativo de niños peruanos en edad escolar, debido al poco interés de los profesionales responsables de su formación, de abordar de manera sistemática y profunda como parte de su tarea científico-pedagógica, da lugar a que no se pueda analizar desde el punto de vista del desarrollo motor las necesidades que presentan los niños, prolongando de este modo la situación existente ${ }^{5}$. Por otro lado, la preeminencia del estudio de la coordinación motora en cuanto a su valor pedagógico como columna fundamental para el aprendizaje de una amplia gama de habilidades y, a su valor clínico, por la posibilidad de identificar insuficiencias de coordinación en las respuestas motoras a situaciones que el medio ambiente impone ${ }^{3,16}$, viabilizará la estructuración de programas didáctico-metodológicos adecuados en las escuelas. Es en este contexto que la realización 
del estudio en intervalos etarios, donde los patrones motores adquiridos son estabilizados, refinados y diversificados ${ }^{17,18}$, así como, en realidades socioeconómicas diferenciadas, conllevó a que el propósito del presente estudio sea: analizar la influencia de la edad, género, estatus socio-económico y de los niveles de adiposidad en el desarrollo coordinativo en escolares peruanos de seis a once años de edad.

\section{METODOLOGÍA}

El diseño del estudio fue transversal y descriptivo. Se estudiaron a 4007 niños de ambos sexos, con edades comprendidas entre los seis y los once años (Cuadro 1). Acudían a siete instituciones educativas, de las cuales cinco son de gestión estatal y dos de gestión privada; todas ubicadas en cuatro distritos del cono este de la ciudad de Lima (LuriganchoChosica, Chaclacayo, Ate-Vitarte y La Molina). Estas instituciones fueron seleccionadas intencionalmente de acuerdo con las facilidades otorgadas para realizar las observaciones, las cuales se realizaron entre los meses de setiembre del 2004 y julio del 2005. En todos los casos se solicitó la autorización de los padres o tutores y el consentimiento individual de cada niño, además del permiso otorgado por autoridades de las instituciones educativas participantes en el estudio.

Cuadro 1: Frecuencia absoluta y relativa de la en función de la edad y en función de la edad y sexo.

\begin{tabular}{ccccc}
\hline & \multicolumn{2}{c}{ Masculino } & \multicolumn{2}{c}{ Feme nino } \\
\cline { 2 - 5 } Edad & $\mathrm{n}$ & $\%$ & $\mathrm{n}$ & $\%$ \\
\hline 6 & 227 & 10,7 & 213 & 11,3 \\
7 & 296 & 14,0 & 270 & 14,3 \\
8 & 340 & 16,1 & 310 & 16,3 \\
9 & 372 & 17,5 & 321 & 17,0 \\
10 & 410 & 19,4 & 345 & 18,3 \\
11 & 473 & 22,3 & 430 & 22,8 \\
\hline Tot al: & 2118 & & 1889 &
\end{tabular}

La CoM fue evaluada a través del Test de Coordinación Corporal para Niños $(\mathrm{KTK})^{6}$, que está constituida de cuatro pruebas: equilibrio en marcha hacia la retaguardia (ER), saltos laterales (SL), saltos monopedales (SM) y transposición lateral (TL), a partir de los cuales se determina un índice global designado como cociente motor (CM). El CM que es establecido a partir de los resultados de las cuatro pruebas de la batería KTK y permite clasificar a los niños según su nivel de desarrollo coordinativo en una de las cinco categorías, no fue considerado para nuestro análisis. Los valores normativos así como los valores de corte para las diferentes categorías fueron establecidos para la población alemana hace cuatro décadas atrás, por lo que su utilización en la población peruana no es adecuada, pues la realidad peruana responde a un país en vías de desarrollo. En lugar de este cociente, se utilizó la sumatoria de las cuatro pruebas que conforman la batería KTK, proporcionando cada una de ellas los mismos pesos en los diferentes intervalos etarios así como en los dos sexos.

La medida de los pliegues de adiposidad subcutánea tricipital, subescapular y pantorrilla medial fue obtenida de acuerdo con el protocolo ISAK ${ }^{19}$. Se realizó la suma de los tres pliegues de adiposidad, luego se dividió a los sujetos en tres grupos distintos en función de la distribución porcentual de los resultados: hasta el 33,3\% (grupo de adiposidad reducida), entre el $33,4 \%$ y $66,5 \%$ (grupo de adiposidad media) y a partir del 66,6\% (grupo de adiposidad elevada). La transformación logarítmica fue un procedimiento necesario para el análisis de los valores de los pliegues de adiposidad subcutánea con la finalidad de cumplir con el requisito de la normalidad de las distribuciones. Los resultados de fiabilidad, estimados a partir del coeficiente de correlación intraclase $(R)$ en los equipos de evaluadores en el estudio piloto, fueron de 0,88 (ER), 0,90 (SL), 0,80 (SM) y 0,91 (TL); mientras que para los pliegues de adiposidad subcutánea fueron de 0,88 (TRI), 0,87 (SUB) y 0,78 (PAN).

Para la caracterización del estatus socioeconómico (ESE) de los escolares, se utilizó como criterio la escuela donde cursaban estudios. Este criterio es válido sobre todo para ser utilizado en países en vías de desarrollo ${ }^{20}$. Con un nivel socio-económico elevado (ESEE) fueron clasificados los estudiantes de escuelas privadas, con nivel socio-económico medio (ESEM) los de las escuelas estatales ubicadas en la zona urbana de cada distrito, y con nivel socioeconómico bajo (ESEB) los pertenecientes a escuelas de zonas urbano-marginales.

Se realizó un análisis exploratorio de los datos con la finalidad de evaluar la normalidad de la distribución, identificar la presencia de "outliers", y obtener un cuadro descriptivo de las variables observadas. Para caracterizar los niveles de desarrollo coordinativo e identificar las diferencias en función de la edad y sexo, serán efectuadas con el recurso al ANOVA con dos factores. Para cuantificar los efectos de la edad, sexo, ESE y niveles de adiposidad subcutánea en los valores parciales y globales de la CoM, se recurrió a modelos de regresión lineal múltiple. El nivel de significancia se mantuvo en $5 \%$. Todos los cálculos fueron realizados haciendo uso del programa estadístico SPSS 15.0.

\section{RESULTADOS}

Los detalles de las variables independientes se exponen en el Cuadro 2. De los 4007 escolares evaluados, el $47 \%$ y el $53 \%$ pertenecen al sexo femenino y masculino, respectivamente. Forman parte del grupo de ESE alto el $24 \%$ de escolares, mientras el 49 y $27 \%$ a los grupos medio y bajo, respectivamente. De los evaluados, presentaban adiposidad elevada el $31 \%$, adiposidad media el $35 \%$ y adiposidad reducida el $34 \%$.

En el Cuadro 3 se presentan las medidas descriptivas básicas de los resultados obtenidos por niños de ambos sexos en los diferentes intervalos etarios de las pruebas de la batería KTK. Se comprueba el incremento de los valores medios de cada prueba y 
Cuadro 2. Características de las variables de estudio.

\begin{tabular}{lrc}
\hline Variables & $\mathbf{N}^{\mathbf{0}}$ & $\%$ \\
\hline Sexo & & \\
$\quad$ Niñas & 1889 & 47,1 \\
$\quad$ Niños & 2118 & 52,9 \\
Edad (años) & & \\
$\quad$ Seis & 440 & 11,1 \\
Siete & 566 & 14,1 \\
Ocho & 650 & 16,2 \\
Nueve & 693 & 17,3 \\
Diez & 755 & 18,8 \\
Once & 903 & 22,5 \\
Estatus Socio-económico & & \\
Alto & 963 & 24,1 \\
Medio & 1977 & 49,3 \\
$\quad$ Bajo & 1067 & 26,6 \\
Nivel de Adiposidad & & \\
Elevada & 954 & 31,2 \\
Media & 1059 & 34,5 \\
Reducida & 1053 & 34,3 \\
\hline
\end{tabular}

de la sumatoria de éstas a lo largo de la edad, en ambos sexos. La magnitud de las desviaciones estándar es baja en las pruebas de TL y SL en ambos grupos, lo que indica cierta homogeneidad de los resultados individuales en torno a los valores medios. De otro lado, en las pruebas de ER y SM los valores de las desviaciones estándar en torno a las medias nos llevan a referir la existencia de una variabilidad interindividual en ambos sexos.

En el Gráfico 1 se representa el comportamiento de los valores medios ( \pm error padrón) de las pruebas de la batería KTK, así como se expone los resultados del ANOVA de dos factores en las diferencias entre los sexos y de los diferentes intervalos etarios en cada prueba del KTK. En todos los intervalos etarios, conforme se aprecia en los resultados del ANOVA II, se verifica diferencias estadísticamente significativas en las pruebas de SM y TL a favor de los niños, sin embargo en ER los resultados favorecen a las niñas. En la prueba de SL no se demostraron diferencias estadísticamente significativas. En cuatro pruebas de la batería KTK y en ambos sexos se comprueba un incremento estadísticamente significativo a lo largo de la edad. En las pruebas de ER y SM no se verificaron una interacción significativa.

Respecto a los resultados de la sumatoria de las cuatro pruebas de la batería KTK, se comprobó que existe a lo largo de la edad y entre ambos sexos diferencias significativas en los valores medios, resultando de la misma manera significativa la interacción entre ambos factores (Gráfico 2).

En los cuadros 4 y 5, se presentan para cada prueba de la batería KTK, así como para la sumatoria de las cuatro pruebas del KTK, los determinantes incluidos en el modelo de regresión que mejor explican la variación en los niveles de desarrollo coordinativo de los niños. En el modelo para cada componente de la batería KTK, el orden de inclusión de los predictores está de acuerdo con los valores de coeficiente de regresión estandarizados. A partir del análisis de regresión lineal múltiple (Cuadro 4), se presentan los siguientes resultados: se confirma lo hallado en el ANOVA II en relación con la edad, que es un determinante significativo en las cuatro pruebas de la batería KTK. Su influencia mayor se observa en la prueba de SM, donde por cada año cronológico que se incrementa, el número de saltos aumenta en 6.28 (IC95\%: 6.05-6.52) puntos. Lo mismo acontece con el sexo, donde los niños relativamente a las niñas muestran un incremento de 6.00 (IC95\%: 5.21, 6.79) puntos en la prueba de SM; en ER los resultados
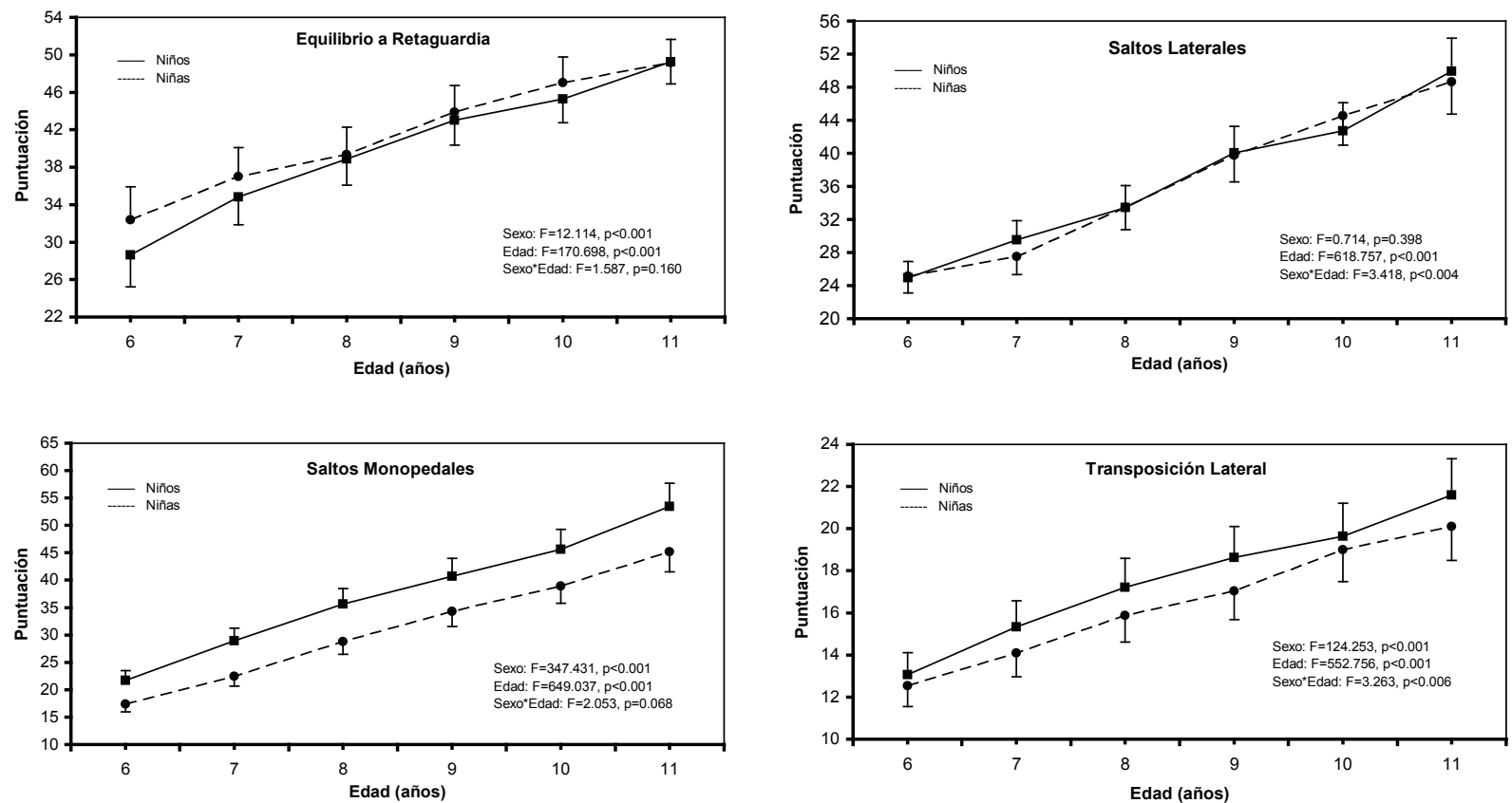

Gráfico 1. Comportamiento de las medias ( \pm error padrón) y resultados del ANOVA II en las diferencias entre los sexos e intervalos etarios en cada prueba del KTK. 
Cuadro 3. Resultados ( $\mathrm{M} \pm$ de) por edad y sexo, de cada una y de la sumatoria de las cuatro pruebas de la batería KTK.

\begin{tabular}{|c|c|c|c|c|c|c|c|c|}
\hline \multirow{2}{*}{$\begin{array}{c}\text { Batería } \\
\text { KTK } \\
\end{array}$} & \multirow[b]{2}{*}{ Sexo } & & \multicolumn{6}{|c|}{ Edad (años) } \\
\hline & & & 6 & 7 & 8 & 9 & 10 & 11 \\
\hline \multicolumn{9}{|l|}{ ER } \\
\hline & M & $M \pm d e$ & $28,63 \pm 12,68$ & $34,83 \pm 13,56$ & $38,87 \pm 13,19$ & $43,02 \pm 12,95$ & $45,30 \pm 13,97$ & $49,25 \pm 12,57$ \\
\hline & $\mathrm{F}$ & $\mathrm{M} \pm \mathrm{de}$ & $32,38 \pm 13,15$ & $36,98 \pm 13,47$ & $39,36 \pm 12,93$ & $43,87 \pm 13,43$ & $47,01 \pm 12,90$ & $49,17 \pm 12,20$ \\
\hline \multicolumn{9}{|l|}{ SL } \\
\hline & $M$ & $\mathrm{M} \pm \mathrm{de}$ & $24,92 \pm 6,65$ & $29,51 \pm 7,18$ & $33,43 \pm 8,76$ & $40,07 \pm 10,05$ & $42,71 \pm 11,18$ & $49,95 \pm 10,80$ \\
\hline & $\mathrm{F}$ & $M \pm d e$ & $25,14 \pm 6,38$ & $27,51 \pm 6,70$ & $33,46 \pm 8,77$ & $39,73 \pm 10,02$ & $44,54 \pm 10,98$ & $48,63 \pm 10,46$ \\
\hline \multicolumn{9}{|l|}{ SM } \\
\hline & M & $M \pm d e$ & $21,72 \pm 9,97$ & $28,94 \pm 10,66$ & $35,61 \pm 11,34$ & $40,70 \pm 11,02$ & $45,64 \pm 12,02$ & $53,44 \pm 12,13$ \\
\hline & $\mathrm{F}$ & $\mathrm{M} \pm \mathrm{de}$ & $17,39 \pm 7,88$ & $22,44 \pm 8,94$ & $28,77 \pm 8,94$ & $34,26 \pm 10,22$ & $38,89 \pm 10,93$ & $45,16 \pm 11,60$ \\
\hline \multicolumn{9}{|l|}{ TL } \\
\hline & M & $M \pm d e$ & $13,06 \pm 2,85$ & $15,34 \pm 3,06$ & $17,22 \pm 2,91$ & $18,62 \pm 2,96$ & $19,63 \pm 3,62$ & $21,60 \pm 3,77$ \\
\hline & $\mathrm{F}$ & $M \pm d e$ & $12,55 \pm 2,70$ & $14,09 \pm 2,92$ & $15,88 \pm 2,81$ & $17,05 \pm 2,71$ & $19,00 \pm 3,40$ & $20,09 \pm 3,05$ \\
\hline \multicolumn{9}{|c|}{$\sum 4$ pruebas } \\
\hline & $M$ & $M \pm d e$ & $88,33 \pm 24,96$ & $108,63 \pm 25,41$ & $125,14 \pm 26,96$ & $142,41 \pm 27,21$ & $153,28 \pm 30,45$ & $174,24 \pm 28,62$ \\
\hline & $\mathrm{F}$ & $\mathrm{M} \pm \mathrm{de}$ & $87,46 \pm 22,19$ & $101,03 \pm 23,76$ & $117,47 \pm 23,46$ & $134,91 \pm 27,10$ & $149,43 \pm 28,35$ & $163,06 \pm 26,18$ \\
\hline
\end{tabular}

revelan que los niños tienen -1.97 puntos (IC95\%: $-2.83,-1.11$ ). En cuanto al estatus socio-económico, los resultados expresan que los niños pertenecientes al ESE alto y medio en relación con los de ESE bajo manifiestan valores más altos en las pruebas de SL 5.30 (IC95\%: 4.28, 6.32), 4.11 (IC95\%: 3.24, 4.98) puntos, respectivamente. El nivel de adiposidad es la determinante que presenta efecto negativo en las cuatro pruebas, es decir, los niños que tienen una adiposidad elevada disminuyen su puntuación en 6.21 (IC95\%: $7.45,-4.97)$ y 6.06 (IC95\%: $-7.20,-4.93)$ relativamente a los niños de adiposidad reducida en las pruebas de ER y SM, respectivamente.

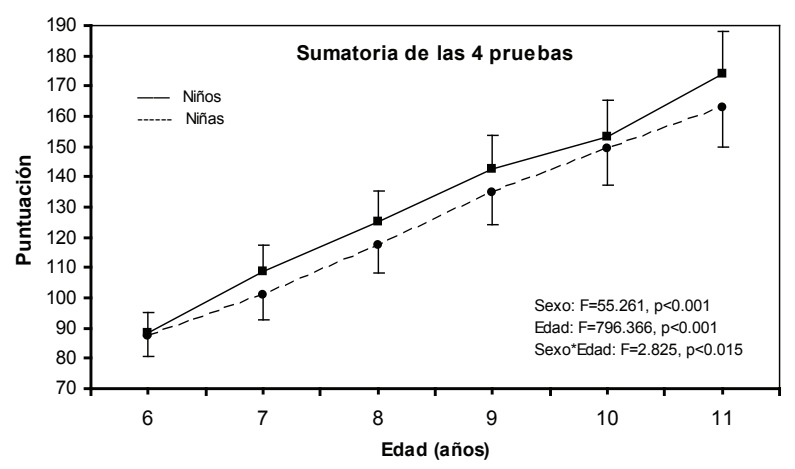

Gráfico 2. Comportamiento de las medias ( \pm error padrón) y resultados del ANOVA II en la sumatoria de las cuatro pruebas de la batería KTK.

Por otro lado, en la sumatoria de las cuatro pruebas de la batería KTK, el predictor que presenta mayor efecto es la edad, con un incremento de 17.01 (IC95\%: 16.46, 17.57) puntos a medida que se transita de un escalón etario a otro. El nivel de adiposidad elevada es un factor negativo, pues los niños que pertenecen a este grupo disminuyen en 16.19 puntos (IC95\%: -18.88, -13.51) en relación con los niños con un nivel de adiposidad baja. Los niños relativamente a las niñas presentan un incremento de 5.27 puntos (IC95\%: $3.40,7.14)$. Formar parte del grupo de ESE medio y alto representa un acrecentamiento de 3.84 (IC95\%: 1.46, 6.22) y 3.43 (IC95\%: 0.62, 6.24) puntos en comparación con los niños de estatus socio-económico bajo.

\section{DISCUSIÓN}

La presente investigación aporta información sobre las características de los niveles de desarrollo coordinativo en ambos sexos y en los diferentes intervalos. De la misma forma, proporciona evidencias sobre las particularidades de los efectos de algunos factores asociados al desarrollo de la capacidad coordinativa corporal de escolares peruanos. Los resultados del ANOVA II confirman que a lo largo de los seis escalones etarios y en ambos sexos existe un aumento significativo de los valores medios del desempeño coordinativo, tal como ya habían sido verificados en estudios realizados por Kiphard e Schilling ${ }^{6}$, Willimczik ${ }^{7}$, Gomes ${ }^{5}$ y Maia y Lopes ${ }^{4,10}$. En cuanto a las diferencias del desempeño coordinativo entre niños y niñas, los resultados encontrados expresan la existencia de diferencias significativas en las pruebas de SM y TL a favor de los niños, en tanto que en ER benefician a las niñas. En la prueba de saltos laterales (SL) no se verifican diferencias estadísticamente significativas, similar a lo encontrado por Maia y Lopes ${ }^{4,10}$. Los incrementos encontrados en nuestro estudio a lo largo de la edad, tienen una justificación en el proceso de crecimiento y maduración en la que están inmersos los niños. También presentan un papel importante las experiencias motoras 
Cuadro 4. Principales determinantes del nivel de coordinación motora gruesa de los niños. Resultados del modelo de regresión múltiple en las cuatro pruebas de la batería KTK.

\begin{tabular}{|c|c|c|c|c|c|c|c|c|}
\hline $\begin{array}{l}\text { Batería } \\
\text { KTK }\end{array}$ & Modelo & $\begin{array}{c}\text { Coeficientes } \\
\text { No estandarizados }\end{array}$ & $\begin{array}{c}\text { Coeficientes } \\
\text { Estandarizados }\end{array}$ & $\begin{array}{l}\text { Error } \\
\text { típico }\end{array}$ & $\mathbf{t}$ & $\mathbf{p}$ & \multicolumn{2}{|c|}{ IC $95 \%$} \\
\hline & Constante & 16,65 & $\longrightarrow$ & 1,25 & 13,27 & $<0,001$ & 14,19 & 19,11 \\
\hline & Edad & 3,84 & 0,47 & 0,13 & 29,44 & $<0,001$ & 3,58 & 4,10 \\
\hline & ESE_alto ${ }^{1}$ & $-6,45$ & $-0,21$ & 0,66 & $-9,74$ & $<0,001$ & $-7,74$ & $-5,15$ \\
\hline \multirow[t]{6}{*}{ ER } & Adip_elevada ${ }^{2}$ & $-6,21$ & $-0,21$ & 0,63 & $-9,81$ & $<0,001$ & $-7,45$ & $-4,97$ \\
\hline & ESE_medio ${ }^{1}$ & $-1,23$ & $-0,09$ & 0,28 & $-4,38$ & $<0,001$ & $-3,55$ & $-1,36$ \\
\hline & Sexo & $-1,97$ & $-0,07$ & 0,44 & $-4,48$ & $<0,001$ & $-2,83$ & $-1,11$ \\
\hline & Adip_media ${ }^{2}$ & $-2,09$ & $-0,07$ & 0,54 & $-3,86$ & $<0,001$ & $-3,15$ & $-1,03$ \\
\hline & Constante & $-9,97$ & $\longrightarrow$ & 0,98 & $-10,14$ & $<0,001$ & $-11,89$ & $-8,04$ \\
\hline & Edad & 5,26 & 0,70 & 0,10 & 51,00 & $<0,001$ & 5,05 & 5,46 \\
\hline \multirow[t]{7}{*}{ SL } & ESEalto $^{1}$ & 5,30 & 0,19 & 0,52 & 10,18 & $<0,001$ & 4,28 & 6,32 \\
\hline & ESEmedio $^{1}$ & 4,11 & 0,16 & 0,44 & 9,29 & $<0,001$ & 3,24 & 4,98 \\
\hline & Adip_elevada ${ }^{2}$ & $-2,98$ & $-0,11$ & 0,49 & $-6,06$ & $<0,001$ & $-3,94$ & $-2,01$ \\
\hline & Adip_media ${ }^{2}$ & $-1,28$ & $-0,05$ & 0,42 & $-3,03$ & $<0,002$ & $-2,11$ & $-0,45$ \\
\hline & Constante & $-21,05$ & - & 1,15 & $-18,29$ & $<0,001$ & $-23,30$ & $-18,79$ \\
\hline & Edad & 6,28 & 0,69 & 0,12 & 52,56 & $<0,001$ & 6,05 & 6,52 \\
\hline & Sexo & 6,00 & 0,19 & 0,40 & 14,89 & $<0,001$ & 5,21 & 6,79 \\
\hline \multirow[t]{7}{*}{ SM } & Adip_elevada ${ }^{2}$ & $-6,06$ & $-0,18$ & 0,58 & $-10,45$ & $<0,001$ & $-7,20$ & $-4,93$ \\
\hline & ESEalto $^{1}$ & 5,13 & 0,15 & 0,61 & 8,46 & $<0,001$ & 3,94 & 6,32 \\
\hline & Adip_media ${ }^{2}$ & $-2,39$ & $-0,07$ & 0,50 & $-4,80$ & $<0,001$ & $-3,36$ & $-1,41$ \\
\hline & ESEmedio ${ }^{1}$ & 1,86 & 0,06 & 0,51 & 3,62 & $<0,001$ & 0,86 & 2,87 \\
\hline & Constante & 3,02 & - & 0,34 & 8,96 & $<0,001$ & 2,36 & 3,69 \\
\hline & Edad & 1,64 & 0,66 & 0,04 & 46,68 & $<0,001$ & 1,57 & 1,71 \\
\hline & Sexo & 0,99 & 0,12 & 0,12 & 8,37 & $<0,001$ & 0,76 & 1,22 \\
\hline \multirow[t]{4}{*}{ TL } & ESEalto $^{1}$ & $-0,51$ & $-0,06$ & 0,18 & $-2,89$ & $<0,001$ & $-0,86$ & $-0,17$ \\
\hline & Adip_elevada ${ }^{2}$ & $-1,02$ & $-0,11$ & 0,17 & $-5,99$ & $<0,001$ & $-1,35$ & $-0,69$ \\
\hline & Adip_media ${ }^{2}$ & $-0,37$ & $-0,04$ & 0,15 & $-2,55$ & $=0,011$ & $-0,66$ & $-0,09$ \\
\hline & ESEmedio ${ }^{1}$ & 0,35 & 0,04 & 0,15 & 2,32 & $=0,021$ & 0,05 & 0,64 \\
\hline
\end{tabular}

${ }^{1}$ Categoría de referencia: ESE bajo.

${ }^{2}$ Categoría de referencia: Nivel de adiposidad reducida.

Cuadro 5. Principales determinantes del nivel de coordinación motora gruesa de los niños. Resultados del modelo de regresión múltiple en la sumatoria de las cuatro pruebas de la batería KTK.

\begin{tabular}{|c|c|c|c|c|c|c|c|c|}
\hline $\begin{array}{c}\text { Batería } \\
\text { KTK }\end{array}$ & Modelo & $\begin{array}{c}\text { Coeficientes } \\
\text { No estandarizados }\end{array}$ & $\begin{array}{c}\text { Coeficientes } \\
\text { Estandarizados }\end{array}$ & $\begin{array}{l}\text { Error } \\
\text { típico }\end{array}$ & $\mathbf{t}$ & $\mathbf{p}$ & \multicolumn{2}{|c|}{ IC 95\% } \\
\hline \multirow{7}{*}{$\sum 4$ pruebas } & Constante & $-11,44$ & $\longrightarrow$ & 2,72 & $-4,21$ & $<0,001$ & $\begin{array}{l}-16,77 \\
\end{array}$ & $-6,11$ \\
\hline & Edad & 17,01 & 0,76 & 0,28 & 60,26 & $<0,001$ & 16,46 & 17,57 \\
\hline & Adip_elevada ${ }^{2}$ & $-16,19$ & $-0,20$ & 1,37 & $-11,82$ & $<0,001$ & $-18,88$ & $-13,51$ \\
\hline & Sexo & 5,27 & 0,07 & 0,95 & 5,54 & $<0,001$ & 3,40 & 7,14 \\
\hline & Adip_media ${ }^{2}$ & $-6,07$ & $-0,08$ & 1,17 & $-5,17$ & $<0,001$ & $-8,37$ & $-3,77$ \\
\hline & ESEmedio $^{1}$ & 3,84 & 0,05 & 1,21 & 3,16 & $<0,002$ & 1,46 & 6,22 \\
\hline & ESEalto $^{1}$ & 3,43 & 0,04 & 1,43 & 2,39 & $=0,017$ & 0,62 & 6,24 \\
\hline
\end{tabular}

${ }^{1}$ Categoría de referencia: ESE bajo.

${ }^{2}$ Categoría de referencia: Nivel de adiposidad reducida.

orientadas pedagógicamente al interior de las escuelas, así como las espontáneas fuera de ellas.

Los valores medios superiores en las niñas en la prueba ER en los diferentes escalones etarios -a excepción de los once años de edad- son similares a los hallados por Kiphard e Schilling ${ }^{6}$ en los primeros cinco escalones etarios; a los de Andrade ${ }^{9}$, a los ocho y nueve años de edad, mientras que sólo a los seis años tienen comportamientos idénticos a lo reportado por Maia y Lopes $^{10}$. En la prueba de TL el comportamiento de los valores medios a favor de los niños son semejantes a los estudios de Portugal, pero diferente a los encontrados por Kiphard e Schilling ${ }^{6}$. En SM también existe similitud en los valores medios superiores a favor de los niños con los datos proporcionados por Gomes $^{5}$, a excepción de la edad de nueve y once años de edad en los estudios de Andrade ${ }^{9}$ y Maia y Lopes $^{10}$. Estos resultados corroboran lo señalado por Willimczik ${ }^{7}$ en relación con la existencia de un padrón de desarrollo específico de cada sexo y no un padrón de desarrollo invariante como sostenía Kiphard e Schilling ${ }^{6}$. Por otro lado, también confirma que las niñas son las más sensibles a las condiciones del medio ambiente y este hecho repercute en su comportamiento motor ${ }^{5}$. Las niñas, por lo general, realizan juegos menos activos y dedican menor tiempo a estas prácticas en comparación con los niños. Los padres son más permisivos con los hijos varones, lo que se traduce en brindarles mayores oportunidades de momentos activos en su quehacer diario. Por otro lado, en las pruebas de ER y SM la interacción resultó no significativa. Se observa un comportamiento similar 
en ambos sexos a lo largo de la edad, es decir que los aumentos de una edad a otra tienen la misma magnitud en ambos sexos. Los resultados de la sumatoria de las cuatro pruebas exhiben diferencias significativas a lo largo de la edad y entre ambos sexos, siendo de la misma manera significativa la interacción entre los predictores sexo y edad.

Del primer modelo de análisis múltiple, destacaremos información valiosa que nos permita dar una lectura diferente a la magnitud de los efectos que tienen los predictores de la edad, el sexo, la pertenencia a un determinado estatus socio-económico y/o presentar un determinado nivel de adiposidad en los valores que reflejan el desarrollo de la coordinación corporal de niños peruanos. La edad es el determinante principal en todas las pruebas de la batería KTK, así como en la sumatoria de ellas. Con base en los coeficientes de regresión, se constata que su mayor influencia se expresa en las pruebas de SM y SL, en las cuales, por cada año de edad adicional, su efecto significa presentar un incremento de 6.28 y 5.26 puntos, respectivamente; siendo en ER de 3.84 puntos y de solo 1.64 en la prueba de TL. Estos resultados se corresponden con lo expuesto en el sentido de que el nivel coordinativo presenta un desarrollo característico a lo largo de la edad, como fue corroborado por distintos estudios realizados en Alemania ${ }^{6,7}$ y Portugal ${ }^{4,5,9,10}$. Si tomamos en cuenta la sumatoria de las cuatro pruebas, la influencia sería de 17.01 puntos por cada incremento del escalón etario, observándose un mayor incremento en los niños de 17.18 puntos frente a 15.12 puntos de las niñas. El mayor incremento se presenta de los diez para los once años de edad en los niños (20.96), y de los ocho para los nueve años de edad en las niñas (17.44). En relación con el sexo, los coeficientes de regresión demuestran que la mayor diferencia a favor de los niños se observa en la prueba de SM con seis puntos, en menor proporción en TL de sólo 0.99 puntos en comparación con las niñas.

El estatus socio-económico tiene un papel importante en las pruebas de SL y SM; relativamente a los niños pertenecientes al grupo de ESE bajo, aquellos que son parte del ESE alto, presentan cinco puntos más en dichas pruebas. Por otro lado, en la prueba de ER, los niños pertenecientes a la categoría de ESE bajo tienen mejor desempeño en comparación a la de ESE alto con puntaje superior en 6.45. En la prueba de TL, los de ESE bajo presentan un desempeño ligeramente superior a los del ESE alto en medio punto; y con los del ESE medio, la diferencia es inexistente. El pertenecer a un estrato socio-económico determinado de por sí ya establece diferencias en el desempeño coordinativo de los niños, como se expresa en los resultados encontrados, donde en las pruebas que requieren mayor participación de la musculatura de las extremidades inferiores (SL y SM) los niños de ESE alto tienen mejores resultados, siendo este grupo el que tiene mejores condiciones de vida. Por otro lado, los niños de ESE bajo que, en nuestra muestra corresponde al $27 \%$, y presentan mejores desempeños en la prueba de ER, son aquellos que desde edades tempranas van adaptando su comportamiento motor a una serie de estímulos ambientales desfavorables, como por ejemplo, desplazarse a pie permanentemente, superar obstáculos de tránsito, jugar en las calles, etcétera, favoreciendo un mayor desarrollo del equilibrio dinámico.

Cuando se analizó la influencia del nivel de adiposidad subcutánea en el desarrollo coordinativo de los niños, se tomó como referencia a la categoría de adiposidad reducida. En las cuatro pruebas se verifica que los escolares (niños y niñas) con adiposidad elevada y media presentan un desempeño inferior relativamente a los escolares con adiposidad reducida. Es en la prueba de ER y SM donde se observa en los escolares con adiposidad elevada un desarrollo coordinativo inferior en seis puntos; mientras en los escolares con adiposidad media la magnitud es menor en promedio dos puntos más bajo en comparación con los que presentan adiposidad reducida. De hecho, la realización de estas dos pruebas exige el desplazamiento horizontal y vertical del centro de gravedad que puede penalizar a los escolares más pesados. Una menor diferencia se constata en las pruebas de SL y TL, siendo dicha diferencia entre uno y tres puntos menos en quienes pertenecen al grupo con adiposidad elevada o media. Maia y Lopes $^{13}$ encontraron en escolares portugueses un decrecimiento ligero de las puntuaciones medias en cada prueba del KTK a medida que el IMC aumentaba, siendo más marcada esta diferencia en la prueba de SM. Un incremento significativo en la prueba de saltos laterales sólo en los niños con peso normal y no así en aquellos con sobrepeso y obesidad pertenecientes a dos tipos de escuela (escuelas con programa de de prevención que combina educación para la salud y actividad física y otras sin programa) fue verificado por Graff'12 en escolares alemanes. Estas evidencias muestran un círculo vicioso que se instala en la vida diaria de los niños: un estilo de vida inactivo conduce a generar un déficit motor, que a su vez consolida hábitos sedentarios adquiridos, dando lugar al desarrollo de sobrepeso y obesidad.

Al presentar un $65,7 \%$ de niños peruanos un nivel de adiposidad elevada y media, refleja los niveles bajos de desarrollo coordinativo que presentan en los diferentes escalones etarios. Una adiposidad subcutánea incrementada es una expresión de los cambios del estilo de vida, de hábitos alimentarios inadecuados y, por otro lado, con el acrecentamiento del peso corporal existe una disminución de actividades físicas, por ende de experiencias motoras que asegure el refinamiento y diversificación en la combinación de las habilidades en padrones secuenciales cada vez más complejos ${ }^{18}$. Es de esperar, entonces, que en un porcentaje importante de niños no se refleje mejoras sustanciales en la capacidad motora a pesar de participar en clases de Educación Física y de actividades deportivas dentro y fuera de las escuelas, mientras no se aborde de manera integral los hechos resaltados en el estudio.

Cuando se comparan los perfiles del presente 

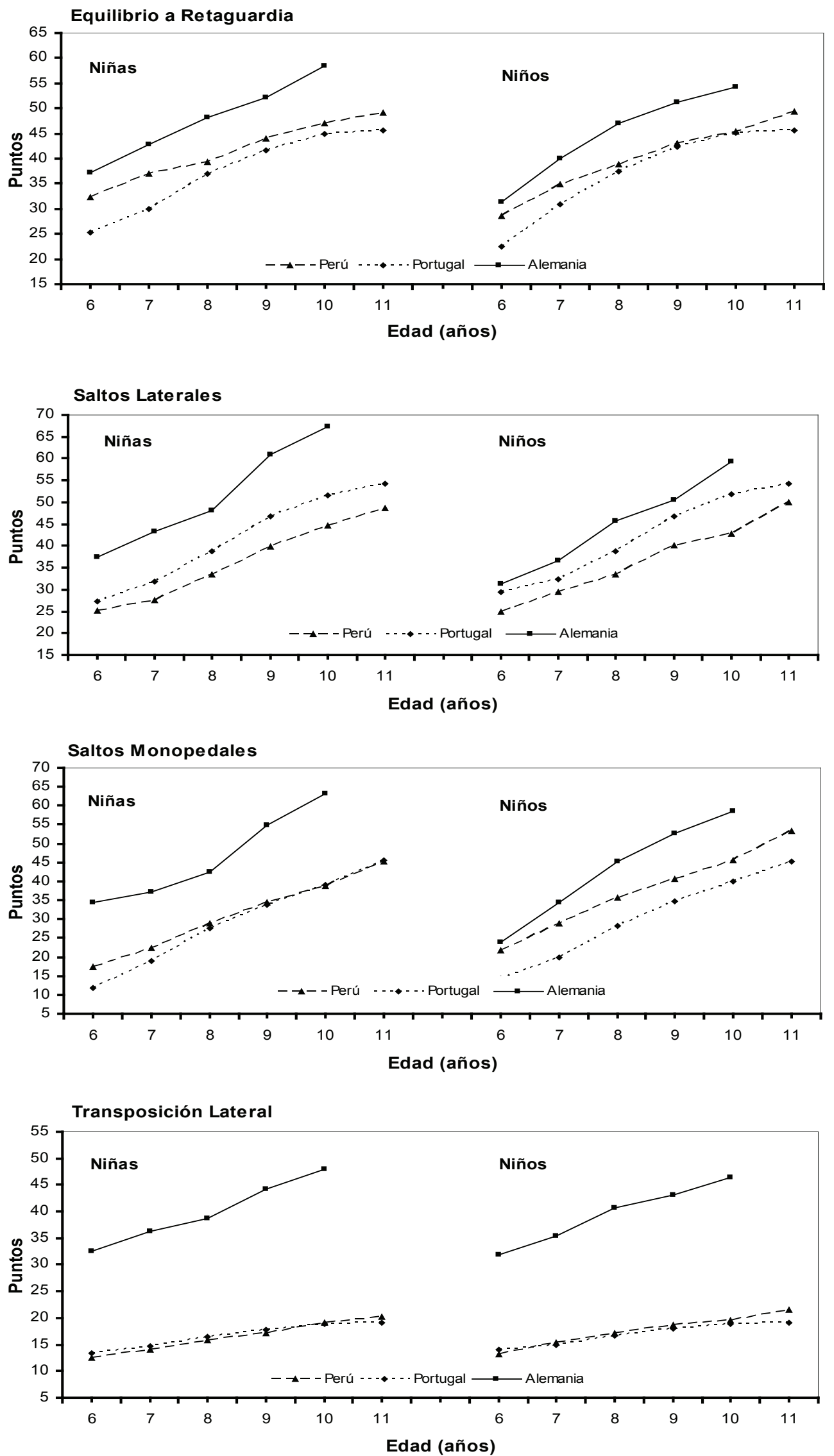

Gráfico 3. Comparación de las trayectorias de los valores medios de las cuatro pruebas de la batería KTK en función de la edad y sexo de escolares peruanos con escolares de otros países (Alemania y Portugal). 
estudio con los resultados encontrados en los estudios de Alemania ${ }^{6}$ y Portugal' ${ }^{10}$ (Gráfico 3 ), se verifican diferencias de nivel y de forma en todos los grupos etarios. Valores inferiores en todas las pruebas en relación con los de niños alemanes en ambos sexos. Debemos destacar en la prueba de TL la diferencia en más de la mitad entre los valores medios que presentan los niños alemanes de ambos sexos en relación con sus pares peruanos y alemanes.

Eran previsibles estas diferencias a favor de los niños alemanes que forman parte de los países desarrollados, que brindan mejores condiciones de vida a su población, y donde las experiencias motoras orientadas pedagógicamente desde edades tempranas están aseguradas. Es decir que los niños alemanes experimentaban otra variedad de actividades motoras y no sólo las espontáneas.

Por otro lado, en la prueba de ER se observa una moderada superioridad en ambos sexos de los escolares peruanos relativamente a los portugueses. En la prueba de SL se constata que los mejores desempeños corresponden a los niños portugueses. En SM sólo se distingue diferencias en el sexo masculino favorables a escolares peruanos. En la prueba de TL, los valores son semejantes en los dos sexos. Estas diferencias advertidas significan que los factores ambientales tienen un rol concluyente que va a imprimir un sello indeleble en el desarrollo coordinativo de los niños. Presentar un estilo de vida y un patrón alimentario inadecuado o pertenecer a un estrato socio-económico que le genere desventajas dentro de su proceso de crecimiento y desarrollo serán factores que ocasionarán insuficiencias en su desarrollo coordinativo.

\section{CONCLUSIÓN}

De acuerdo con los propósitos del estudio y según los resultados encontrados, se concluye que: (1) en todas las pruebas de la batería KTK, en ambos sexos y a lo largo de la edad, existe un incremento significativo de los valores medios, lo cual expresa la presencia de un desarrollo coordinativo específico para cada sexo, así como refleja la plasticidad del desarrollo de la coordinación motora; (2) el nivel de adiposidad presenta una influencia significativa en los resultados de cada una de las pruebas y en la sumatoria de ellas, teniendo, los niños con adiposidad elevada, rendimientos inferiores en comparación con los de adiposidad reducida; (3) el estatus socio-económico no es un predictor concluyente en las cuatro pruebas, siendo su influencia positiva en las pruebas de saltos laterales y saltos monopedales, mientras es negativa en las de equilibrio a retaguardia y transposición lateral.

Los hallazgos disponibles reafirman la necesidad de implementar programas de Educación Física y de iniciación deportiva debidamente planificados, clases estructuradas didáctica y metodológicamente apropiadas a cada edad, procedimientos de evaluación y control de los logros de los alumnos, que permita un desarrollo coordinativo adecuado como parte de una formación integral en los niños.

\section{REFERÊNCIAS BIBLIOGRÁFICAS}

1. Lopes V, Maia JA, Silva R, Seabra A, Morais F. Estudo do nível de desenvolvimento da coordenação motora da população escolar (6 a 10 anos de idade) da Região Autónoma dos Açores. Rev Port Ci Des 2003; 3(1):4760.

2. Smits-Engelsman BCM, Henderson S, Michels C. The assessment of children with Developmental Coordination Disorders in the Netherlands: The relationship between the Movement Assessment Battery for Children and the KÖrperkoordinations Test für Kinder. Hum Mov Sci 1998; 17:699-709.

3. Kiphard EJ. Insuficiencias de movimiento y de coordinación en la edad de la escuela primaria. Buenos Aires: Editorial Kapelusz; 1976.

4. Maia JAR, Lopes VP. Estudo do crescimento somatico, aptitud física, actividade física e capacidade de coordinaçao corporal de crianças do $1^{\circ} \mathrm{Ciclo}$ do Ensino Básico da Region Autónoma de Açores. Edit. Multitema, Portugal; 2002.

5. Gomes MPBB. Coordenação, aptidão física e variáveis do envolvimento. Estudo em crianças do $1^{\circ}$ ciclo de ensino de duas freguesias do concelho de Matosinhos. [Tese de doutoramento]. Porto: Universidade de Porto; 1996.

6. Kiphard, B. J., y Schilling, F. Körperkoordinations Test für Kinder. Beltz Test GmbH, Weinheim: 1974.

7. Willimczik, K. Development of motor control capability (body coordination) of 6-to 10-year-old children: Results of a Longitudinal Study. In M. Ostyn; G. Beunen; J. Simons,editors. Kinanthropometry II. Baltimore: University Park Press; 1980. p. 328-346.

8. Koreeda K, Kobayashi Y. A study on the body coordination ability of clumsy children in elementary school. 13th International Symposium Adapted Physical Activity. Vienna, Austria; 2001.

9. Andrade MJLA. Coordenação motora. Estudo em crianças do ensino básico na Região Autónoma da Madeira. [Dissertação de mestrado]. Porto: Universidade de Porto; 1996.

10. Maia JA, Lopes VP. Crescimento e desenvolvimento de crianças e jovens Açorianos. O que pais, professores, pediatras e nutricionistas gostariam de saber. Universidade de Porto; 2007.

11. Graf C, Koch B, Kretschmann-Kandel E, Falkowski G, Christ $\mathrm{H}$, Coburger $\mathrm{S}$, et al. Correlation between BMI, leisure habits and motor abilities in childhood (CHILTProject). Int J Obes 2004;28,22-26

12. Graf C, Koch B, Falkowski G, Jouck S, Christ H, Stauenmaier K, et al. Effects of a school-based intervention on BMI and motor abilities in childhood. J Sports Sci Med 2005; 4,291-299.

13. Maia JAR, Lopes VP. Um Olhar Sobre Crianças e Jovens da Região Autónoma dos Açores. Implicações para a Educação Física, Desportos e Saúde. U. de Porto; 2003.

14. Silva GAS. Análise da coordenação ampla (grossa) em crianças de 7 a 10 anos. [Dissertação de Mestrado em Educação Física]. São Paulo: U. de São Paulo; 1989.

15. Bustamante A, Caballero L, Quispe S, Rodríguez G, Enciso N, Rosas C, et al. Crecimiento somático, coordinación motora y actividad física en escolares del Nivel Primario: Implicancias para la Educación Física, Deporte y Salud. Lima, 2005.

16. Meinel K. Motricidade II: O Desenvolvimento Motor do Ser Humano. Ao Livro Técnico S/A, col. Educação Física, Rio de Janeiro, 1984. 
17. Tani G. Educação física na pré-escola e nas quatro primeiras séries do ensino de primeiro grau: uma abordagem de desenvolvimento I. Revista Kinesis 1987; 3:19-41.

18. Gallahue D. Understanding motor development in children. New York: Wiley, 1982.

19. Norton K, Whittingham N, Carter L, Kerr D, Gore C, Marfell-Jones M. Measurement Techniques in Anthropometry. En: Norton K, Olds T, editors. Anthropometrica. Sydney: University of New South Wales Press; 1996. p. 45-85
20. Prista A, Marques A, Maia J. Relationship Between Physical Activity, Socioeconomic Status, and Physical Fitness of 8-15-Year-Old Youth From Mozambique. Am J Hum Biol 1997; 9:449-457.

\section{Agradecimientos}

Los autores agradecen a la Fundación para la Ciencia y la Tecnología de Portugal por la beca de manutención SFRH/BM/15929/. Extendemos nuestro agradecimiento a los estudiantes de Educación Física de la UNE que participaron en la recolección de datos.

\section{Endereço para correspondência}

Alcibíades Bustamante Valdivia

Recebido em 30/05/07

Universidade do Porto

Revisado em 08/07/07

Faculdade de Desporto

Aprovado em 16/07/07

Laboratório de Cineantropometria e Estatística Aplicada

Rua Plácido Costa 91, 4200.450

Porto, Portugal

E-mail: huanta2609@yahoo.es 